\title{
Strong Subgraph Connectivity of Digraphs
}

\author{
Yuefang Sun ${ }^{1} \cdot$ Gregory Gutin ${ }^{2}$
}

Received: 11 June 2019/Revised: 25 February 2021 / Accepted: 3 March 2021 /

Published online: 18 March 2021

(C) The Author(s) 2021

\section{Abstract}

Let $D=(V, A)$ be a digraph of order $n, S$ a subset of $V$ of size $k$ and $2 \leq k \leq n$. A strong subgraph $H$ of $D$ is called an $S$-strong subgraph if $S \subseteq V(H)$. A pair of $S$ strong subgraphs $D_{1}$ and $D_{2}$ are said to be arc-disjoint if $A\left(D_{1}\right) \cap A\left(D_{2}\right)=\emptyset$. A pair of arc-disjoint $S$-strong subgraphs $D_{1}$ and $D_{2}$ are said to be internally disjoint if $V\left(D_{1}\right) \cap V\left(D_{2}\right)=S$. Let $\kappa_{S}(D)$ (resp. $\lambda_{S}(D)$ ) be the maximum number of internally disjoint (resp. arc-disjoint) $S$-strong subgraphs in $D$. The strong subgraph $k$-connectivity is defined as

$$
\kappa_{k}(D)=\min \left\{\kappa_{S}(D)|S \subseteq V,| S \mid=k\right\} .
$$

As a natural counterpart of the strong subgraph $k$-connectivity, we introduce the concept of strong subgraph $k$-arc-connectivity which is defined as

$$
\lambda_{k}(D)=\min \left\{\lambda_{S}(D)|S \subseteq V(D),| S \mid=k\right\} .
$$

A digraph $D=(V, A)$ is called minimally strong subgraph $(k, \ell)$-(arc-)connected if $\kappa_{k}(D) \geq \ell\left(\right.$ resp. $\left.\lambda_{k}(D) \geq \ell\right)$ but for any arc $e \in A, \kappa_{k}(D-e) \leq \ell-1$ (resp. $\left.\lambda_{k}(D-e) \leq \ell-1\right)$. In this paper, we first give complexity results for $\lambda_{k}(D)$, then obtain some sharp bounds for the parameters $\kappa_{k}(D)$ and $\lambda_{k}(D)$. Finally, minimally strong subgraph $(k, \ell)$-connected digraphs and minimally strong subgraph $(k, \ell)$-arcconnected digraphs are studied.

Keywords Directed graph connectivity · Strong subgraph connectivity · Strong subgraph arc connectivity · Generalized connectivity · Arc-disjoint subgraph decomposition

Gregory Gutin

g.gutin@ rhul.ac.uk

1 School of Mathematics and Statistics, Ningbo University, Zhejiang 315211, People's Republic of China

2 Department of Computer Science, Royal Holloway, University of London, Egham, Surrey TW20 0EX, UK 


\section{Introduction}

The generalized $k$-connectivity $\kappa_{k}(G)$ of a graph $G=(V, E)$ was introduced by Hager [8] in $1985(2 \leq k \leq|V|)$. For a graph $G=(V, E)$ and a set $S \subseteq V$ of at least two vertices, an $S$-Steiner tree or, simply, an $S$-tree is a subgraph $T$ of $G$ which is a tree with $S \subseteq V(T)$. Two $S$-trees $T_{1}$ and $T_{2}$ are said to be internally disjoint if $E\left(T_{1}\right) \cap E\left(T_{2}\right)=\emptyset$ and $V\left(T_{1}\right) \cap V\left(T_{2}\right)=S$. The generalized local connectivity $\kappa_{S}(G)$ is the maximum number of internally disjoint $S$-trees in $G$. For an integer $k$ with $2 \leq k \leq n$, the generalized $k$-connectivity is defined as

$$
\kappa_{k}(G)=\min \left\{\kappa_{S}(G)|S \subseteq V(G),| S \mid=k\right\} .
$$

Observe that $\kappa_{2}(G)=\kappa(G)$. If $G$ is disconnected and vertices of $S$ are placed in different connectivity components, we have $\kappa_{S}(G)=0$. Thus, $\kappa_{k}(G)=0$ for a disconnected graph $G$. Generalized connectivity of graphs has become an established area in graph theory, see a recent monograph [9] by Li and Mao on generalized connectivity of undirected graphs.

To extend generalized $k$-connectivity to directed graphs, Sun et al. [13] observed that in the definition of $\kappa_{S}(G)$, one can replace "an $S$-tree" by "a connected subgraph of $G$ containing $S$ ". Therefore, Sun et al. [13] defined strong subgraph $k$ connectivity by replacing "connected" with "strongly connected" (or, simply, "strong") as follows. Let $D=(V, A)$ be a digraph of order $n, S$ a subset of $V$ of size $k$ and $2 \leq k \leq n$. A subgraph $H$ of $D$ is called an $S$-strong subgraph if $S \subseteq V(H)$. A pair of $S$-strong subgraphs $D_{1}$ and $D_{2}$ are said to be arc-disjoint if $A\left(D_{1}\right) \cap A\left(D_{2}\right)=\emptyset$. A pair of arc-disjoint $S$-strong subgraphs $D_{1}$ and $D_{2}$ are said to be internally disjoint if $V\left(D_{1}\right) \cap V\left(D_{2}\right)=S$. Let $\kappa_{S}(D)$ be the maximum number of internally disjoint $S$-strong subgraphs in $D$. The strong subgraph $k$-connectivity [13] is defined as

$$
\kappa_{k}(D)=\min \left\{\kappa_{S}(D)|S \subseteq V(D),| S \mid=k\right\} .
$$

By definition, $\kappa_{2}(D)=0$ if $D$ is not strong.

As a natural counterpart of the strong subgraph $k$-connectivity, we now introduce the concept of strong subgraph $k$-arc-connectivity. Let $\lambda_{S}(D)$ be the maximum number of arc-disjoint $S$-strong digraphs in $D$. The strong subgraph $k$-arcconnectivity is defined as

$$
\lambda_{k}(D)=\min \left\{\lambda_{S}(D)|S \subseteq V(D),| S \mid=k\right\} .
$$

By definition, $\lambda_{2}(D)=0$ if $D$ is not strong.

For a digraph $D$, its reverse $D^{\text {rev }}$ is a digraph with same vertex set and such that $x y \in A\left(D^{\mathrm{rev}}\right)$ if and only if $y x \in A(D)$. A digraph $D$ is symmetric if $D^{\mathrm{rev}}=D$. In other words, a symmetric digraph $D$ can be obtained from its underlying undirected graph $G$ by replacing each edge of $G$ with the corresponding arcs of both directions, that is, $D=\overleftrightarrow{G}$.

The strong subgraph $k$-(arc-)connectivity is not only a natural extension of the concept of generalized $k$-(edge-)connectivity, but also relates to important problems 
in graph theory. For $k=2, \kappa_{2}(\overleftrightarrow{G})=\kappa(G)[13]$ and $\lambda_{2}(\overleftrightarrow{G})=\lambda(G)$ (Theorem 3.6). Hence, $\kappa_{k}(D)$ and $\lambda_{k}(D)$ could be seen as generalizations of connectivity and edgeconnectivity of undirected graphs, respectively. For $k=n, \kappa_{n}(D)=\lambda_{n}(D)$ is the maximum number of arc-disjoint spanning strong subgraphs of $D$. Moreover, since $\kappa_{S}(G)$ and $\lambda_{S}(G)$ are the number of internally disjoint and arc-disjoint strong subgraphs containing a given set $S$, respectively, these parameters are relevant to the problem of finding the maximum number of strong spanning arc-disjoint subgraphs in a digraph studied, e.g., in [3-5, 12].

In what follows, $n$ will denote the number of vertices of the digraph under consideration.

A digraph $D=(V(D), A(D))$ is called minimally strong subgraph $(k, \ell)$ connected if $\kappa_{k}(D) \geq \ell$ but for any arc $e \in A(D), \kappa_{k}(D-e) \leq \ell-1$. Similarly, a digraph $D=(V(D), A(D))$ is called minimally strong subgraph $(k, \ell)$-arc-connected if $\lambda_{k}(D) \geq \ell$ but for any arc $e \in A(D), \lambda_{k}(D-e) \leq \ell-1$.

A 2-cycle $x y x$ of a strong digraph $D$ is called a bridge if $D-\{x y, y x\}$ is disconnected. Thus, a bridge corresponds to a bridge in the underlying undirected graph of $D$. An orientation of a digraph $D$ is a digraph obtained from $D$ by deleting an arc in each 2-cycle of $D$. A digraph $D$ is semicomplete if for every distinct $x, y \in V(D)$ at least one of the arcs $x y, y x$ is in $D$. A digraph $D$ is $k$-regular if the inand out-degree of every vertex of $D$ is equal to $k$. We refer the readers to [2] for graph theoretical notation and terminology not given here.

Let $k \geq 2$ and $\ell \geq 2$ be fixed integers. By reduction from the Directed 2-LinKage problem, Sun et al. [13] proved that deciding whether $\kappa_{S}(D) \geq \ell$ is NP-complete for a $k$-subset $S$ of $V(D)$. Thomassen [14] showed that for every positive integer $p$ there are digraphs which are strongly $p$-connected, but which contain a pair of vertices not belonging to the same cycle. This implies that for every positive integer $p$ there are strongly $p$-connected digraphs $D$ such that $\kappa_{2}(D)=1$ [13].

The above negative results motivate studying strong subgraph $k$-connectivity for special classes of digraphs. In Sun et al. [13], showed that the problem of deciding whether $\kappa_{k}(D) \geq \ell$ for every semicomplete digraphs is polynomial-time solvable for fixed $k$ and $\ell$. The main tool used in their proof is a recent Directed $k$-LinKage theorem of Chudnovsky, Scott and Seymour [7]. Sun et al. [13] showed that for any connected graph $G$, the parameter $\kappa_{2}(\overleftrightarrow{G})$ can be computed in polynomial time. This result is best possible in the following sense. Let $D$ be a symmetric digraph and $k \geq 3$ a fixed integer. Then it is NP-complete to decide whether $\kappa_{S}(D) \geq \ell$ for $S \subseteq V(D)$ with $|S|=k$ [13]. Let $D$ be a strong digraph with $n$ vertices. Sun et al. [13] proved that $1 \leq \kappa_{k}(D) \leq n-1$ for $2 \leq k \leq n$. The bounds are sharp; Sun et al. [13] also characterized those digraphs $D$ for which $\kappa_{k}(D)$ attains the upper bound. The main tool used in their proof is a Hamiltonian cycle decomposition theorem of Tillson [15].

In this paper, we prove that for fixed integers $k, \ell \geq 2$, the problem of deciding whether $\lambda_{S}(D) \geq \ell$ is NP-complete for a digraph $D$ and a set $S \subseteq V(D)$ of size $k$. This result is proved in Sect. 3 using the corresponding result for $\kappa_{S}(D)$ proved in [13]. In the same section, we also consider classes of digraphs. We characterize when $\lambda_{k}(D) \geq 2,2 \leq k \leq n$, for both semicomplete and symmetric digraphs $D$ of 
order $n$. The characterizations imply that the problem of deciding whether $\lambda_{k}(D) \geq 2$ is polynomial-time solvable for both semicomplete and symmetric digraphs. For fixed $\ell \geq 3$ and $k \geq 2$, the complexity of deciding whether $\lambda_{k}(D) \geq \ell$ remains an open problem for both semicomplete and symmetric digraphs. It was proved in [13] that for fixed $k, \ell \geq 2$ the problem of deciding whether $\kappa_{k}(D) \geq \ell$ is polynomial-time solvable for both semicomplete and symmetric digraphs, but it appears that the approaches to prove the two results cannot be used for $\lambda_{k}(D)$. In fact, we would not be surprised if the $\lambda_{k}(D) \geq \ell$ problem turns out to be NP-complete at least for one of the two classes of digraphs.

In Sect. 4, we first give sharp upper bounds for the parameters $\kappa_{k}(D)$ and $\lambda_{k}(D)$ in terms of classical connectivity. Then we get some lower and upper bounds for the parameter $\lambda_{k}(D)$ including a lower bound whose analog for $\kappa_{k}(D)$ does not hold as well as Nordhaus-Gaddum type bounds.

In Sect. 5, we characterize minimally strong subgraph $(2, n-2)$-connected digraphs and minimally strong subgraph $(2, n-2)$-arc-connected digraphs. Also, we bound the sizes of minimally strong subgraph $(2, n-2)$-connected digraphs.

We conclude the paper in Sect. 6 by discussing open problems.

\section{Preliminaries}

Let us start this section from observations that can be easily verified using definitions of $\lambda_{k}(D)$ and $\kappa_{k}(D)$. Note that the first inequality of the following inequalities (2) can be found in [13].

Proposition 2.1 Let $D$ be a digraph of order $n$, and let $k \geq 2$ be an integer. Then

$$
\lambda_{k+1}(D) \leq \lambda_{k}(D) \text { for every } k \leq n-1
$$

For a spanning subgraph $D^{\prime}$ of $D$, we have

$$
\begin{gathered}
\kappa_{k}\left(D^{\prime}\right) \leq \kappa_{k}(D), \lambda_{k}\left(D^{\prime}\right) \leq \lambda_{k}(D) \\
\kappa_{k}(D) \leq \lambda_{k}(D) \leq \min \left\{\delta^{+}(D), \delta^{-}(D)\right\}
\end{gathered}
$$

The inequality (1) means that the parameter $\lambda_{k}$ has a monotonically nonincreasing with respect to $k$. However, this property may not hold for $\kappa_{k}$, that is, $\kappa_{n}(D) \leq \kappa_{n-1}(D) \leq \cdots \leq \kappa_{3}(D) \leq \kappa_{2}(D)=\kappa(D)$ may not be true. Consider the following example: Let $D$ be a digraph obtained from two copies $D_{1}$ and $D_{2}$ of the complete digraph $\overleftrightarrow{K}_{t}(t \geq 4)$ by identifying one vertex in each of them. Clearly, $D$ is a strong digraph with a cut vertex, say $u$. For $2 \leq k \leq 2 t-2$, let $S$ be a subset of $V(D) \backslash\{u\}$ with $|S|=k$ such that $S \cap V\left(D_{i}\right) \neq \emptyset$ for every $i \in\{1,2\}$. Since each $S$ strong subgraph must contain $u$, we have $\kappa_{k}(D) \leq 1$, furthermore, we deduce that $\kappa_{k}(D)=1$ for $2 \leq k \leq 2 t-2$. Let $G_{i}$ be the underlying undirected graph of $D_{i}$ for $i \in\{1,2\}$. Each $G_{i}$ contains $\left\lfloor\frac{t}{2}\right\rfloor$ edge-disjoint spanning trees, say $T_{i, j}\left(1 \leq j \leq\left\lfloor\frac{t}{2}\right\rfloor\right)$, since $G_{i}$ is a complete graph of order $t$ (see, e.g., (3.1) in [10]). Now in $D$, let $H_{j}$ be a 
subgraph of $D$ obtained from the tree $T_{j}$ which is the union of $T_{1, j}$ and $T_{2, j}$ by replacing each edge with two arcs of the opposite directions. Clearly, these subgraphs are strong, spanning and arc-disjoint. Hence, $\kappa_{2 t-1}(D) \geq\left\lfloor\frac{t}{2}\right\rfloor>1=$ $\kappa_{k}(D)$ for $2 \leq k \leq 2 t-2$.

We will use the following decomposition theorem by Tillson.

Theorem 2.2 [15] The arcs of $\overleftrightarrow{K}_{n}$ can be decomposed into Hamiltonian cycles if and only if $n \neq 4,6$.

\section{Complexity}

Yeo proved that it is an NP-complete problem to decide whether a 2-regular digraph has two arc-disjoint hamiltonian cycles (see, e.g., Theorem 6.6 in [5]). Thus, the problem of deciding whether $\lambda_{n}(D) \geq 2$ is NP-complete, where $n$ is the order of $D$. We will extend this result in Theorem 3.1.

Let $D$ be a digraph and let $s_{1}, s_{2}, \ldots, s_{k}, t_{1}, t_{2}, \ldots, t_{k}$ be a collection of not necessarily distinct vertices of $D$. A weak $k$-linkage from $\left(s_{1}, s_{2}, \ldots, s_{k}\right)$ to $\left(t_{1}, t_{2}, \ldots, t_{k}\right)$ is a collection of $k$ arc-disjoint paths $P_{1}, \ldots, P_{k}$ such that $P_{i}$ is an $\left(s_{i}, t_{i}\right)$-path for each $i \in[k]$. A digraph $D=(V, A)$ is weakly $k$-linked if it contains a weak $k$-linkage from $\left(s_{1}, s_{2}, \ldots, s_{k}\right)$ to $\left(t_{1}, t_{2}, \ldots, t_{k}\right)$ for every choice of (not necessarily distinct) vertices $s_{1}, \ldots, s_{k}, t_{1}, \ldots, t_{k}$. The WEAK $k$-LINKAGE PROBLEM is the following. Given a digraph $D=(V, A)$ and distinct vertices $x_{1}, x_{2}, \ldots, x_{k}, y_{1}, y_{2}, \ldots, y_{k}$; decide whether $D$ contains $k$ arc-disjoint paths $P_{1}, \ldots, P_{k}$ such that $P_{i}$ is an $\left(x_{i}, y_{i}\right)$-path. The problem is well-known to be NPcomplete already for $k=2$ [2].

Theorem 3.1 Let $k \geq 2$ and $\ell \geq 2$ be fixed integers. Let $D$ be a digraph and $S \subseteq$ $V(D)$ with $|S|=k$. The problem of deciding whether $\lambda_{S}(D) \geq \ell$ is NP-complete.

Proof Clearly, the problem is in NP. We will show that it is NP-hard using a reduction similar to that in Theorem 2.1 of [13]. Let us first deal with the case of $\ell=2$ and $k=2$. Consider the digraph $D^{\prime}$ used in the proof of Theorem 2.1 of [13] (see Fig. 1), where $D$ is an arbitrary digraph, $x, y$ are vertices not in $D$, and $t_{1} x, x s_{1}, t_{2} y, y s_{2}, x s_{2}, s_{2} x, y t_{1}, t_{1} y$ are additional arcs. To construct a new digraph $D^{\prime \prime}$ from $D^{\prime}$, replace every vertex $u$ of $D$ by two vertices $u^{-}$and $u^{+}$such that $u^{-} u^{+}$is an arc in $D^{\prime \prime}$ and for every $u v \in A(D)$ add an arc $u^{+} v^{-}$to $D^{\prime \prime}$. Also, for $z \in\{x, y\}$, for every arc $z u$ in $D^{\prime}$ add an arc $z u^{-}$to $D^{\prime \prime}$ and for every arc $u z$ add an $\operatorname{arc} u^{+} z$ to $D^{\prime \prime}$.

Fig. 1 The digraph $D^{\prime}$

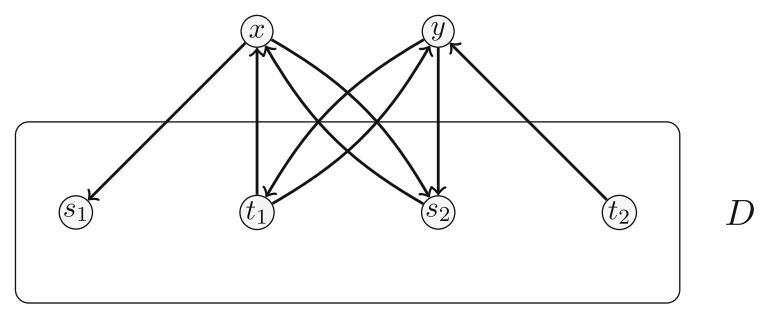


Let $S=\{x, y\}$. It was proved in Theorem 2.1 of [13] that $\kappa_{S}\left(D^{\prime}\right) \geq 2$ if and only if there are vertex-disjoint paths from $s_{1}$ to $t_{1}$ and from $s_{2}$ to $t_{2}$. It follows from this result and definition of $D^{\prime \prime}$ that $\lambda_{S}\left(D^{\prime \prime}\right) \geq 2$ if and only if there are arc-disjoint paths from $s_{1}^{-}$to $t_{1}^{+}$and from $s_{2}^{-}$to $t_{2}^{+}$. Since the WEAK 2-LINKAGE PROBLEM is NP-complete, we conclude that the problem of deciding whether $\lambda_{S}\left(D^{\prime \prime}\right) \geq 2$ is NP-hard.

Now let us consider the case of $\ell \geq 3$ and $k=2$. Add to $D^{\prime \prime} \ell-2$ copies of the 2cycle $x y x$ and subdivide the arcs of every copy to avoid parallel arcs. Let us denote the new digraph by $D^{\prime \prime \prime}$. Similarly to that in Theorem 2.1 of [13], we can show that $\lambda_{S}\left(D^{\prime \prime \prime}\right) \geq \ell$ if and only if $\lambda_{S}\left(D^{\prime \prime}\right) \geq 2$.

It remains to consider the case of $\ell \geq 2$ and $k \geq 3$. Add to $D^{\prime \prime \prime}$ (where $D^{\prime \prime \prime}=D^{\prime \prime}$ for $\ell=2) k-2$ new vertices $x_{1}, \ldots, x_{k-2}$ and arcs of $\ell 2$-cycles $x x_{i} x$ for each $i \in[k-2]$. Subdivide the new arcs to avoid parallel arcs. Denote the obtained digraph by $D^{\prime \prime \prime \prime}$. Let $S=\left\{x, y, x_{1}, \ldots, x_{k-2}\right\}$. Similarly to that in Theorem 2.1 of [13], we can show that $\lambda_{S}\left(D^{\prime \prime \prime \prime}\right) \geq \ell$ if and only if $\lambda_{S}\left(D^{\prime \prime}\right) \geq 2$.

Bang-Jensen and Yeo [5] conjectured the following:

Conjecture 1 For every $\lambda \geq 2$ there is a finite set $\mathcal{S}_{\lambda}$ of digraphs such that a $\lambda$-arcstrong semicomplete digraph $D$ contains $\lambda$ arc-disjoint spanning strong subgraphs unless $D \in \mathcal{S}_{\lambda}$.

Bang-Jensen and Yeo [5] proved the conjecture for $\lambda=2$ by showing that $\left|\mathcal{S}_{2}\right|=$ 1 and describing the unique digraph $S_{4}$ of $\mathcal{S}_{2}$ of order 4 . Now we have the following characterization:

Theorem 3.2 For a semicomplete digraph $D$, of order $n$ and an integer $k$ such that $2 \leq k \leq n, \lambda_{k}(D) \geq 2$ if and only if $D$ is 2-arc-strong and the following does not hold: $D \cong S_{4}$ and $k=4$.

Proof We first consider the direction "only if". Suppose that $D$ is not a 2-arc-strong and $x y \in A(D)$ such that $D-x y$ is not strong. Thus, for $S=\{x, y\}$ we have $\lambda_{S}(D)=$ 1. Hence $\lambda_{2}(D)=1$ and by (1) $\lambda_{k}(D)=1$ for each $k, 2 \leq k \leq n$. Furthermore, by the result of Bang-Jensen and Yeo, the following does not hold: $D \cong S_{4}$ and $k=4$.

We next prove the direction "if". If $D$ is 2 -arc-strong and $D \neq S_{4}$, then $D$ contains two arc-disjoint spanning strong subgraphs by the result of Bang-Jensen and Yeo, that is, $\lambda_{n}(D) \geq 2$. Furthermore, we have $\lambda_{k}(D) \geq 2$ for all $2 \leq k \leq n$ by (1). Now we consider the case that $D \cong S_{4}$. Let $S$ be any subset of $V(D)$ with $|S|=3$; by symmetry of $S_{4}$ it suffices to assume that $S=\left\{v_{1}, v_{2}, v_{3}\right\}$ (see Fig. 2). Let $D_{1}$ be the cycle $v_{1}, v_{2}, v_{3}, v_{1}$ and $D_{2}$ be subgraph of $D$ with $A\left(D_{2}\right)=A(D) \backslash A\left(D_{1}\right)$. It can be easily checked that both $D_{1}$ and $D_{2}$ are $S$-strong subgraphs, so $\lambda_{3}(D) \geq 2$. Furthermore by (1), we have $\lambda_{2}(D) \geq 2$.

Now we turn our attention to symmetric digraphs. We start from characterizing symmetric digraphs $D$ with $\lambda_{k}(D) \geq 2$, an analog of Theorem 3.2. To prove it we will use the following result of Boesch and Tindell [6] translated from the language of mixed graphs to that of digraphs. 
Fig. 2 Digraph $S_{4}$

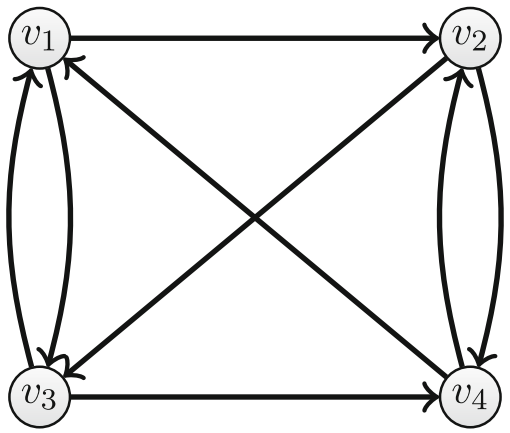

Theorem 3.3 A strong digraph $D$ has a strong orientation if and only if D has no bridge.

Here is our characterization.

Theorem 3.4 For a strong symmetric digraph $D$ of order $n$ and an integer $k$ such that $2 \leq k \leq n, \lambda_{k}(D) \geq 2$ if and only if $D$ has no bridge.

Proof Let $D$ have no bridge. Then, by Theorem 3.3, $D$ has a strong orientation $H$. Since $D$ is symmetric, $H^{\mathrm{rev}}$ is another orientation of $D$. Clearly, $H^{\mathrm{rev}}$ is strong and hence $\lambda_{k}(D) \geq 2$.

Suppose that $D$ has a bridge $x y x$. Choose a set $S$ of size $k$ such that $\{x, y\} \subseteq S$ and observe that any strong subgraph of $D$ containing vertices $x$ and $y$ must include both $x y$ and $y x$. Thus, $\lambda_{S}(D)=1$ and $\lambda_{k}(D)=1$.

Theorems 3.2 and 3.4 imply the following complexity result, which we believe to be extendable from $\ell=2$ to any natural $\ell$.

Corollary 3.5 The problem of deciding whether $\lambda_{k}(D) \geq 2$ is polynomial-time solvable if $D$ is either semicomplete or symmetric digraph of order $n$ and $2 \leq k \leq n$.

Now we give a lower bound on $\lambda_{k}(D)$ for symmetric digraphs $D$.

Theorem 3.6 For every graph $G$, we have

$$
\lambda_{k}(\overleftrightarrow{G}) \geq \lambda_{k}(G)
$$

Moreover, this bound is sharp. In particular, we have $\lambda_{2}(\overleftrightarrow{G})=\lambda_{2}(G)$.

Proof We may assume that $G$ is a connected graph. Let $S=\{x, y\}$, where $x, y$ are distinct vertices of $\overleftrightarrow{G}$. Observe that $\lambda_{S}(G) \geq \lambda_{S}(\overleftrightarrow{G})$. Indeed, let $p=\lambda_{S}(\overleftrightarrow{G})$ and let $D_{1}, \ldots, D_{p}$ be arc-disjoint $S$-strong subgraphs of $\overleftrightarrow{G}$. Thus, by choosing a path from $x$ to $y$ in each $D_{i}$, we obtain $p$ arc-disjoint paths from $x$ to $y$, which correspond to $p$ arcdisjoint paths between $x$ and $y$ in $G$. Thus, $\lambda(G)=\lambda_{2}(G) \geq \lambda_{2}(\overleftrightarrow{G})$.

We now consider the general $k$. Let $\lambda_{S}(\overleftrightarrow{G})=\lambda_{k}(\overleftrightarrow{G})$ for some $S \subseteq V(\overleftrightarrow{G})$ with $|S|=k$. We know that there are at least $\lambda_{k}(G)$ edge-disjoint trees containing $S$ in $G$, say $T_{i}\left(i \in\left[\lambda_{k}(G)\right]\right)$. For each $i \in\left[\lambda_{k}(G)\right]$, we can obtain a strong subgraph 
containing $S$, say $D_{i}$, in $\overleftrightarrow{G}$ by replacing each edge of $T_{i}$ with the corresponding arcs of both directions. Clearly, any two such subgraphs are arc-disjoint, so we have $\lambda_{k}(\overleftrightarrow{G})=\lambda_{S}(\overleftrightarrow{G}) \geq \lambda_{k}(G)$, and we also have $\lambda_{2}(\overleftrightarrow{G})=\lambda_{2}(G)=\lambda(G)$

For the sharpness of the bound, consider the tree $T$ with order $n$. Clearly, we have $\lambda_{k}(T)=1$. Furthermore, $1 \leq \lambda_{k}(\overleftrightarrow{T}) \leq \min \left\{\delta^{+}(D), \delta^{-}(D)\right\}=1$ by Inequality (3).

Note that for the case that $3 \leq k \leq n$, the equality $\lambda_{k}(\overleftrightarrow{G})=\lambda_{k}(G)$ does not always hold. For example, consider the cycle $C_{n}$ of order $n$; it is not hard to check that $\lambda_{k}\left(\overleftrightarrow{C}_{n}\right)=2$, but $\lambda_{k}\left(C_{n}\right)=1$

Theorem 3.6 immediately implies the next result, which follows from the fact that $\lambda(G)$ can be computed in polynomial time.

Corollary 3.7 For a symmetric digraph $D, \lambda_{2}(D)$ can be computed in polynomial time.

\section{Sharp bounds of $\kappa_{k}(D)$ and $\lambda_{k}(D)$}

To prove a new bound on $\kappa_{k}(D)$ in Theorem 4.2, we will use the following result of Sun et al. [13].

Theorem 4.1 Let $2 \leq k \leq n$. For a strong digraph $D$ of order $n$, we have

$$
1 \leq \kappa_{k}(D) \leq n-1
$$

Moreover, both bounds are sharp, and the upper bound holds if and only if $D \cong \overleftrightarrow{K}_{n}$, $2 \leq k \leq n$ and $k \notin\{4,6\}$.

The following result concerns the relation between $\kappa_{k}(D)\left(\right.$ resp. $\left.\lambda_{k}(D)\right)$ and $\kappa(D)$ (resp. $\lambda(D))$.

Theorem 4.2 Let $k \in\{2, \ldots, n\}$. The following assertions hold:

(i) For $n \geq \kappa(D)+k$, we have $\kappa_{k}(D) \leq \kappa(D)$;

(ii) $\quad \lambda_{k}(D) \leq \lambda(D)$. Moreover, both bounds are sharp.

Proof Part (i). For $k=2$, assume that $\kappa(D)=\kappa(x, y)$ for some $\{x, y\} \subseteq V(D)$. It follows from the strong subgraph connectivity definition that $\kappa_{\{x, y\}}(D) \leq \kappa(x, y)$, so $\kappa_{2}(D) \leq \kappa_{\{x, y\}}(D) \leq \kappa(x, y)=\kappa(D)$.

We now consider the case of $k \geq 3$. If $\kappa(D)=n-1$, then we have $\kappa_{k}(D) \leq n-$ $1=\kappa(D)$ by Theorem 4.1. If $\kappa(D)=n-2$, then there are two vertices, say $u$ and $v$, such that $u v \notin A(D)$. So we have $\kappa_{k}(D) \leq n-2=\kappa(D)$ by Theorem 4.1. If $1 \leq \kappa(D) \leq n-3$, then there exists a $\kappa(D)$-vertex cut, say $Q$, for two vertices $u, v$ in $D$ such that there is no $u-v$ path in $D-Q$. Let $S=\{u, v\} \cup S^{\prime}$ where $S^{\prime} \subseteq$ $V(D) \backslash(Q \cup\{u, v\})$ and $\left|S^{\prime}\right|=k-2$. Since $u$ and $v$ are in different strong components of $D-Q$, any $S$-strong subgraph in $D$ must contain a vertex in $Q$. By the definition of $\kappa_{S}(D)$ and $\kappa_{k}(D)$, we have $\kappa_{k}(D) \leq \kappa_{S}(D) \leq|Q|=\kappa(D)$.

For the sharpness of the bound, consider the following digraph $D$. Let $D$ be a 
symmetric digraph whose underlying undirected graph is $K_{k} \bigvee \bar{K}_{n-k}(n \geq 3 k)$, i.e. the graph obtained from disjoint graphs $K_{k}$ and $\bar{K}_{n-k}$ by adding all edges between the vertices in $K_{k}$ and $\bar{K}_{n-k}$.

Let $V(D)=W \cup U$, where $W=V\left(K_{k}\right)=\left\{w_{i} \mid 1 \leq i \leq k\right\}$ and $U=V\left(\bar{K}_{n-k}\right)=$ $\left\{u_{j} \mid 1 \leq j \leq n-k\right\}$. Let $S$ be any $k$-subset of vertices of $V(D)$ such that $|S \cap U|=s$ $(s \leq k)$ and $|S \cap W|=k-s$. Without loss of generality, let $w_{i} \in S$ for $1 \leq i \leq k-s$ and $u_{j} \in S$ for $1 \leq j \leq s$. For $1 \leq i \leq k-s$, let $D_{i}$ be the symmetric subgraph of $D$ whose underlying undirected graph is the tree $T_{i}$ with edge set

$$
\left\{w_{i} u_{1}, w_{i} u_{2}, \ldots, w_{i} u_{s}, u_{k+i} w_{1}, u_{k+i} w_{2}, \ldots, u_{k+i} w_{k-s}\right\} .
$$

For $k-s+1 \leq j \leq k$, let $D_{j}$ be the symmetric subgraph of $D$ whose underlying undirected graph is the tree $T_{j}$ with edge set

$$
\left\{w_{j} u_{1}, w_{j} u_{2}, \ldots, w_{j} u_{s}, w_{j} w_{1}, w_{j} w_{2}, \ldots, w_{j} w_{k-s}\right\} .
$$

Observe that $\left\{D_{i} \mid 1 \leq i \leq k-s\right\} \cup\left\{D_{j} \mid k-s+1 \leq j \leq k\right\}$ is a set of $k$ internally disjoint $S$-strong subgraph, so $\kappa_{S}(D) \geq k$, and then $\kappa_{k}(D) \geq k$. Combining this with the bound that $\kappa_{k}(D) \leq \kappa(D)$ and the fact that $\kappa(D) \leq \min \left\{\delta^{+}(D), \delta^{-}(D)\right\}=k$, we can get $\kappa_{k}(D)=\kappa(D)=k$.

Part (ii) Let $A$ be a $\lambda(D)$-arc-cut of $D$, where $1 \leq \lambda(D) \leq n-1$. We choose $S \subseteq V(D)$ such that at least two of these $k$ vertices are in different strong components of $D-A$. Thus, any $S$-strong subgraph in $D$ must contain an arc in $A$. By the definition of $\lambda_{S}(D)$ and $\lambda_{k}(D)$, we have $\lambda_{k}(D) \leq \lambda_{S}(D) \leq|A|=\lambda(D)$.

For the sharpness of the bound, consider the the digraph $D$ in part $(i)$. Recall that $\left\{D_{i} \mid 1 \leq i \leq k\right\}$ is a set of $k$ internally disjoint $S$-strong subgraph, so $\lambda_{S}(D) \geq \kappa_{S}(D) \geq k$, and then $\lambda_{k}(D) \geq k$. Combining this with the bound that $\lambda_{k}(D) \leq \lambda(D)$ and the fact that $\lambda(D) \leq \min \left\{\delta^{+}(D), \delta^{-}(D)\right\}=k$, we can get $\lambda_{k}(D)=\lambda(D)=k$.

Note that the condition " $n \geq \kappa(D)+k$ " in Theorem 4.2 cannot be removed. Consider the example after Proposition 2.1. We have $n=2 t-1<2 t=\kappa(D)+k$ when $k=n$, but now $\kappa_{n}(D)>\kappa(D)$.

In the proof of Theorem 4.1, they used the following result on $\kappa_{k}\left(\overleftrightarrow{K}_{n}\right)$.

Lemma 4.3 [13] For $2 \leq k \leq n$, we have

$$
\kappa_{k}\left(\overleftrightarrow{K}_{n}\right)=\left\{\begin{array}{lc}
n-1, & \text { if } k \notin\{4,6\} \\
n-2, & \text { otherwise }
\end{array}\right.
$$

We can now compute the exact values of $\lambda_{k}\left(\overleftrightarrow{K}_{n}\right)$.

Lemma 4.4 For $2 \leq k \leq n$, we have 


$$
\lambda_{k}\left(\overleftrightarrow{K}_{n}\right)= \begin{cases}n-1, & \text { if } k \notin\{4,6\}, \quad \text { or, } k \in\{4,6\} \text { and } k<n \\ n-2, & \text { if } k=n \in\{4,6\}\end{cases}
$$

Proof For the case that $2 \leq k \leq n$ and $k \notin\{4,6\}$, by (3) and Lemma 4.3, we have $n-1 \leq \kappa_{k}\left(\overleftrightarrow{K}_{n}\right) \leq \lambda_{k}\left(\overleftrightarrow{K}_{n}\right) \leq n-1$. Hence, $\lambda_{k}\left(\overleftrightarrow{K}_{n}\right)=n-1$ and in the following argument we assume that $2 \leq k \leq n$ and $k \in\{4,6\}$.

We first consider the case of $2 \leq k=n$. For $n=4$, since $K_{n}$ contains a Hamiltonian cycle, the two orientations of the cycle imply that $\lambda_{n}\left(\overleftrightarrow{K}_{n}\right) \geq 2=n-2$. To see that there are at most two arc-disjoint strong spanning subgraphs of $\overleftrightarrow{K}_{n}$, suppose that there are three arc-disjoint such subgraphs. Then each such subgraph must have exactly four arcs (as $\left|A\left(\overleftrightarrow{K}_{n}\right)\right|=12$ ), and so all of these three subgraphs are Hamiltonian cycles, which means that the arcs of $\overleftrightarrow{K}_{n}$ can be decomposed into Hamiltonian cycles, a contradiction to Theorem 2.2). Hence, $\lambda_{n}\left(\overleftrightarrow{K}_{n}\right)=n-2$ for $n=4$. Similarly, we can prove that $\lambda_{n}\left(\overleftrightarrow{K}_{n}\right)=n-2$ for $n=6$, as $K_{n}$ contains two edge-disjoint Hamiltonian cycles, and therefore $\overleftrightarrow{K}_{n}$ contains four arc-disjoint Hamiltonian cycles.

We next consider the case of $2 \leq k \leq n-1$. We assume that $k=6$ as the case of $k=4$ can be considered in a similar and simpler way. Let $S \subseteq V\left(\overleftrightarrow{K}_{n}\right)$ be any vertex subset of size six. Let $S=\left\{u_{i} \mid 1 \leq i \leq 6\right\}$ and $V\left(\overleftrightarrow{K}_{n}\right) \backslash S=\left\{v_{j} \mid 1 \leq j \leq n-6\right\}$. Let $D_{1}$ be the cycle $u_{1} u_{2} u_{3} u_{4} u_{5} u_{6} u_{1}$; let $D_{2}=D_{1}^{\text {rev }}$; let $D_{3}$ be the cycle $u_{1} u_{3} u_{6} u_{4} u_{2} u_{5} u_{1}$; let $D_{4}=D_{3}^{\text {rev }}$; let $D_{5}$ be a subgraph of $\overleftrightarrow{K}_{n}$ with vertex set $S \cup\left\{v_{1}\right\}$ and arc set $\left\{u_{1} v_{1}, v_{1} u_{2}, u_{2} u_{6}, u_{6} v_{1}, v_{1} u_{5}, u_{5} u_{3}, u_{3} v_{1}, v_{1} u_{4}, u_{4} u_{1}\right\} ; \quad$ let $D_{6}=D_{5}^{\text {rev }} ; \quad$ for each $x \in\left\{v_{j} \mid 2 \leq j \leq n-6\right\}$, let $D_{x}$ be a subgraph of $\overleftrightarrow{K}_{n}$ with vertex set $S \cup\{x\}$ and arc set $\left\{x u_{i}, u_{i} x \mid 1 \leq i \leq 6\right\}$. Hence, we have $\lambda_{S}(D) \geq n-1$ for any $S \subseteq V\left(\overleftrightarrow{K}_{n}\right)$ with $|S|=6$ and so $\lambda_{k}(D) \geq n-1$. We clearly have $\lambda_{k}(D) \leq n-1$ by (3), then our result holds.

Now we obtain sharp lower and upper bounds for $\lambda_{k}(D)$ for $2 \leq k \leq n$.

Theorem 4.5 Let $2 \leq k \leq n$. For a strong digraph $D$ of order $n$, we have

$$
1 \leq \lambda_{k}(D) \leq n-1
$$

Moreover, both bounds are sharp, and the upper bound holds if and only if $D \cong \overleftrightarrow{K}_{n}$, where $k \notin\{4,6\}$, or, $k \in\{4,6\}$ and $k<n$.

Proof The lower bound is clearly correct by the definition of $\lambda_{k}(D)$, and for the sharpness, a cycle is our desired digraph. The upper bound and its sharpness hold by (2) and Lemma 4.4.

If $D$ is not equal to $\overleftrightarrow{K}_{n}$ then $\delta^{+}(D) \leq n-2$ and by (3) we observe that $\lambda_{k}(D) \leq \delta^{+}(D) \leq n-2$. Therefore, by Lemma 4.4, the upper bound holds if and only if $D \cong \overleftrightarrow{K}_{n}$, where $k \notin\{4,6\}$, or, $k \in\{4,6\}$ and $k<n$. 
Shiloach [11] proved the following:

Theorem 4.6 [11] A digraph $D$ is weakly k-linked if and only if $D$ is $k$-arc-strong.

Using Shiloach's Theorem, we will prove the following lower bound for $\lambda_{k}(D)$. Such a bound does not hold for $\kappa_{k}(D)$ since it was shown in [13] using Thomassen's result in [14] that for every $\ell$ there are digraphs $D$ with $\kappa(D)=\ell$ and $\kappa_{2}(D)=1$.

Proposition 4.7 Let $k \leq \ell=\lambda(D)$. We have $\lambda_{k}(D) \geq\lfloor\ell / k\rfloor$.

Proof Choose an arbitrary vertex set $S=\left\{s_{1}, \ldots, s_{k}\right\}$ of $D$ and let $t=\lfloor\ell / k\rfloor$. By Theorem 4.6, there is a weak $k t$-linkage $L$ from $x_{1}, x_{2}, \ldots, x_{k t}$ to $y_{1}, y_{2}, \ldots, y_{k t}$, where $x_{i}=s_{i \bmod k}$ and $y_{i}=s_{i \bmod k+1}$ and $s_{k+1}=s_{1}$. Note that the paths of $L$ form $t$ arcdisjoint strong subgraphs of $D$ containing $S$.

For a digraph $D=(V(D), A(D))$, the complement digraph, denoted by $D^{c}$, is a digraph with vertex set $V\left(D^{c}\right)=V(D)$ such that $x y \in A\left(D^{c}\right)$ if and only if $x y \notin A(D)$.

Given a graph parameter $f(G)$, the Nordhaus-Gaddum Problem is to determine sharp bounds for (a) $f(G)+f\left(G^{c}\right)$ and (b) $f(G) f\left(G^{c}\right)$, and characterize the extremal graphs. The Nordhaus-Gaddum type relations have received wide attention; see a recent survey paper [1] by Aouchiche and Hansen. Theorem 4.9 concerns such type of a problem for the parameter $\lambda_{k}$. To prove the theorem, we will need the following:

Proposition 4.8 A digraph $D$ with order $n$ is strong if and only if $\lambda_{k}(D) \geq 1$, where $2 \leq k \leq n$.

Proof If $D$ is strong, then for every vertex set $S$ of size $k, D$ has a strong subgraph containing $S$. If $\lambda_{k}(D) \geq 1$, for each vertex set $S$ of size $k$ construct $D_{S}$, a strong subgraph of $D$ containing $S$. The union of all $D_{S}$ is a strong subgraph of $D$ as there are sets $S_{1}, S_{2}, \ldots, S_{p}$ such that the union of $S_{1}, S_{2}, \ldots, S_{p}$ is $V(D)$ and for each $i \in[p-1], D_{S_{i}}$ and $D_{S_{i+1}}$ share a common vertex.

Theorem 4.9 For a digraph $D$ with order $n$, the following assertions hold:

$0 \leq \lambda_{k}(D)+\lambda_{k}\left(D^{c}\right) \leq n-1$. Moreover, both bounds are sharp. In particular, the lower bound holds if and only if $\lambda(D)=\lambda\left(D^{c}\right)=0$.

(ii) $\quad 0 \leq \lambda_{k}(D) \lambda_{k}\left(D^{c}\right) \leq\left(\frac{n-1}{2}\right)^{2}$. Moreover, both bounds are sharp. In particular, the lower bound holds if and only if $\lambda(D)=0$ or $\lambda\left(D^{c}\right)=0$.

Proof We first prove (i). Since $D \cup D^{c}=\overleftrightarrow{K}_{n}$, by definition of $\lambda_{k}$, $\lambda_{k}(D)+\lambda_{k}\left(D^{c}\right) \leq \lambda_{k}\left(\overleftrightarrow{K}_{n}\right)$. Thus, by Lemma 4.4 , the upper bound for the sum $\lambda_{k}(D)+\lambda_{k}\left(D^{c}\right)$ holds. Let $H \cong \overleftrightarrow{K}_{n}$. When $k \notin\{4,6\}$, or, $k \in\{4,6\}$ and $k<n$, by Lemma 4.4 , we have $\lambda_{k}(H)=n-1$ and we clearly have $\lambda_{k}\left(H^{c}\right)=0$, so the upper bound is sharp.

The lower bound is clear. Clearly, the lower bound holds, if and only if $\lambda_{k}(D)=\lambda_{k}\left(D^{c}\right)=0$, if and only if $\lambda(D)=\lambda\left(D^{c}\right)=0$ by Proposition 4.8 .

We now prove (ii). The lower bound is clear, and it holds, if and only if $\lambda_{k}(D)=$ 
0 or $\lambda_{k}\left(D^{c}\right)=0$, if and only if $\lambda(D)=0$ or $\lambda\left(D^{c}\right)=0$ by Proposition 4.8. For the upper bound, we have

$$
\lambda_{k}(D) \lambda_{k}\left(D^{c}\right) \leq\left(\frac{\lambda_{k}(D)+\lambda_{k}\left(D^{c}\right)}{2}\right)^{2} \leq\left(\frac{n-1}{2}\right)^{2} .
$$

Let $H \cong \overleftrightarrow{K}_{n}$ with $n=2 h+1 \geq 7$. By Theorem $2.2, H$ contains $2 h$ arc-disjoint Hamiltonian cycles: $H_{1}, \ldots, H_{2 h}$. Let $D_{1}$ be the union of the former $h$ cycles, and $D_{2}$ be the union of the remaining $h$ cycles. Clearly, $D_{1}^{c}=D_{2}$ and $\lambda_{n}\left(D_{i}\right) \geq h$ and so $\lambda_{k}\left(D_{i}\right) \geq h$ for $1 \leq i \leq 2,2 \leq k \leq n$ by (1). Furthermore, $D_{i}$ is $h$-regular, so $\lambda_{k}\left(D_{i}\right) \leq h$ by (3). Hence, $\lambda_{k}\left(D_{i}\right)=h$ for $1 \leq i \leq 2,2 \leq k \leq n$. Now $\lambda_{k}\left(D_{1}\right) \lambda_{k}\left(D_{1}^{c}\right)=$ $\lambda_{k}\left(D_{1}\right) \lambda_{k}\left(D_{2}\right)=h^{2}=\left(\frac{n-1}{2}\right)^{2}$, so the upper bound is sharp.

\section{Minimally Strong Subgraph $(k, \ell)$-(arc-)connected Digraphs}

In this section, we will first study the minimally strong subgraph $(k, \ell)$-connected digraphs. By the definition of a minimally strong subgraph $(k, \ell)$-connected digraph, we can get the following observation.

Proposition 5.1 A digraph $D$ is minimally strong subgraph $(k, \ell)$-connected if and only if $\kappa_{k}(D)=\ell$ and $\kappa_{k}(D-e)=\ell-1$ for any arc $e \in A(D)$.

Proof The direction "if" is clear by definition, and we only need to prove the direction "only if". Let $D$ be a minimally strong subgraph $(k, \ell)$-connected digraph. By definition, we have $\kappa_{k}(D) \geq \ell$ and $\kappa_{k}(D-e) \leq \ell-1$ for any arc $e \in A(D)$. Then for any set $S \subseteq V(D)$ with $|S|=k$, there is a set $\mathcal{D}$ of $\ell$ internally disjoint $S$-strong subgraphs. As $e$ must belong to one and only one element of $\mathcal{D}$, we are done. D.

A digraph $D$ is minimally strong if $D$ is strong but $D-e$ is not for every arc $e$ of

Proposition 5.2 The following assertions hold:

(i) A digraph $D$ is minimally strong subgraph $(k, 1)$-connected if and only if $D$ is a minimally strong digraph;

(ii) For $k \neq 4,6$, a digraph $D$ is minimally strong subgraph $(k, n-1)$ connected if and only if $D \cong \overleftrightarrow{K}_{n}$.

Proof To prove (i), it suffices to show that a digraph $D$ is strong if and only if $\kappa_{k}(D) \geq 1$. If $D$ is strong, then for every vertex set $S$ of size $k, D$ has an $S$-strong subgraph. If $\kappa_{k}(D) \geq 1$, for each vertex set $S$ of size $k$ construct $D_{S}$, an $S$-strong subgraph of $D$. The union of all $D_{k}$ is a strong subgraph of $D$ as there are sets $S_{1}, S_{2}, \ldots, S_{p}$ such that the union of $S_{1}, S_{2}, \ldots, S_{p}$ is $V(D)$ and for each $i \in[p-1]$, $D_{S_{i}}$ and $D_{S_{i+1}}$ share a common vertex.

Part (ii) follows from Theorem 4.1.

The following result characterizes minimally strong subgraph $(2, n-2)$ connected digraphs. 
Theorem 5.3 A digraph $D$ is minimally strong subgraph $(2, n-2)$-connected if and only if $D$ is a digraph obtained from the complete digraph $\overleftrightarrow{K}_{n}$ by deleting an arc set $M$ such that $\overleftrightarrow{K}_{n}[M]$ is a 3-cycle or a union of $\lfloor n / 2\rfloor$ vertex-disjoint 2-cycles. In particular, we have $f(n, 2, n-2)=n(n-1)-2\lfloor n / 2\rfloor, \quad F(n, 2, n-2)=$ $n(n-1)-3$.

Proof Let $D \cong \overleftrightarrow{K}_{n}-M$ be a digraph obtained from the complete digraph $\overleftrightarrow{K}_{n}$ by deleting an arc set $M$. Let $V(D)=\left\{u_{i} \mid 1 \leq i \leq n\right\}$.

Firstly, we will consider the case that $\overleftrightarrow{K}_{n}[M]$ is a 3 -cycle $u_{1} u_{2} u_{3} u_{1}$. We now prove that $\kappa_{2}(D)=n-2$. By (3), we have $\kappa_{2}(D) \leq \min \left\{\delta^{+}(D), \delta^{-}(D)\right\}=n-2$. Let $S=\{u, v\} \subseteq V(D)$; we just consider the case that $u=u_{1}, v=u_{2}$ since the other cases are similar. Let $D_{1}$ be a subgraph of $D$ with $V\left(D_{1}\right)=\left\{u_{1}, u_{2}, u_{3}\right\}$ and $A\left(D_{1}\right)=\left\{u_{1} u_{3}, u_{3} u_{2}, u_{2} u_{1}\right\}$; for $2 \leq i \leq n-2$, let $D_{i}$ be a subgraph of $D$ with $V\left(D_{i}\right)=\left\{u_{1}, u_{2}, u_{i+2}\right\}$ and $A\left(D_{i}\right)=\left\{u_{1} u_{i+2}, u_{2} u_{i+2}, u_{i+2} u_{1}, u_{i+2} u_{2}\right\}$. Clearly, $\left\{D_{i} \mid\right.$ $1 \leq i \leq n-2\}$ is a set of $n-2$ internally disjoint $S$-strong subgraphs, so $\kappa_{S}(D) \geq n-2$ and $\kappa_{2}(D) \geq n-2$. Hence, $\kappa_{2}(D)=n-2$.

For any $e \in A(D)$, without loss of generality, one of the two digraphs in Fig. 3 is a subgraph of $\overleftrightarrow{K}_{n}[M \cup\{e\}]$, so if the following claim holds, then we must have $\kappa_{2}(D-e) \leq \kappa_{2}\left(D^{\prime}\right) \leq n-3$ by Proposition 4.3 , and so $D$ is minimally strong subgraph $(2, n-2)$-connected. Now it suffices to prove the following claim.

Claim 1 If $\overleftrightarrow{K}_{n}\left[M^{\prime}\right]$ is isomorphic to one of two graphs in Fig. 3, then $\kappa_{2}\left(D^{\prime}\right) \leq n-3$, where $D^{\prime}=\overleftrightarrow{K}_{n}-M^{\prime}$

Proof of Claim 1 We first show that $\kappa_{2}\left(D^{\prime}\right) \leq n-3$ if $M^{\prime}$ is the digraph of Fig. 3a. Let $S=\left\{u_{2}, u_{4}\right\}$; we will prove that $\kappa_{S}\left(D^{\prime}\right) \leq n-3$, and then we are done. Suppose that $\kappa_{S}\left(D^{\prime}\right) \geq n-2$, then there exists a set of $n-2$ internally disjoint $S$-strong subgraphs, say $\left\{D_{i} \mid 1 \leq i \leq n-2\right\}$. If both of the two arcs $u_{2} u_{4}$ and $u_{4} u_{2}$ belong to the same $D_{i}$, say $D_{1}$, then for $2 \leq i \leq n-2$, each $D_{i}$ contains at least one vertex and at most two vertices of $\left\{u_{i} \mid 1 \leq i \leq n, i \neq 2,4\right\}$. Furthermore, there is at most one $D_{i}$, say $D_{2}$, contains (exactly) two vertices of $\left\{u_{i} \mid 1 \leq i \leq n, i \neq 2,4\right\}$. We just consider the case that $u_{1}, u_{3} \in V\left(D_{2}\right)$ since the other cases are similar. In this case, we must have that each vertex of $\left\{u_{i} \mid 5 \leq i \leq n\right\}$ belongs to exactly one digraph from $\left\{D_{i} \mid 3 \leq i \leq n-2\right\}$ and vice versa. However, this is impossible since the vertex set $\left\{u_{2}, u_{4}, u_{5}\right\}$ cannot induce an $S$-strong subgraph of $D^{\prime}$, a contradiction.

So we now assume that each $D_{i}$ contains at most one of $u_{2} u_{4}$ and $u_{4} u_{2}$. Without

Fig. 3 Two graphs for Claim 1

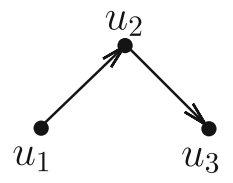

(a)

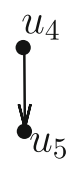
(b) 
loss of generality, we may assume that $u_{2} u_{4} \in A\left(D_{1}\right)$ and $u_{4} u_{2} \in A\left(D_{2}\right)$. In this case, we must have that each vertex of $\left\{u_{i} \mid 1 \leq i \leq n, i \neq 2,4\right\}$ belongs to exactly one digraph from $\left\{D_{i} \mid 1 \leq i \leq n-2\right\}$ and vice versa. However, this is also impossible since the vertex set $\left\{u_{2}, u_{4}, u_{5}\right\}$ cannot induce an $S$-strong subgraph of $D^{\prime}$, a contradiction.

Hence, we have $\kappa_{2}\left(D^{\prime}\right) \leq n-3$ in this case. For the case that $M^{\prime}$ is the digraph of Fig. $3 \mathrm{~b}$, we can choose $S=\left\{u_{2}, u_{3}\right\}$ and prove that $\kappa_{S}\left(D^{\prime}\right) \leq n-3$ with a similar argument, and so $\kappa_{2}\left(D^{\prime}\right) \leq n-3$ in this case. This completes the proof of the claim.

Secondly, we consider the case that $\overleftrightarrow{K}_{n}[M]$ is a union of $\lfloor n / 2\rfloor$ vertex-disjoint 2cycles. Without loss of generality, we may assume that $M=\left\{u_{2 i-1} u_{2 i}, u_{2 i} u_{2 i-1} \mid 1 \leq i \leq\lfloor n / 2\rfloor\right\}$. We just consider the case that $S=$ $\left\{u_{1}, u_{3}\right\}$ since the other cases are similar. In this case, let $D_{1}$ be the subgraph of $D$ with $V\left(D_{1}\right)=\left\{u_{1}, u_{3}\right\}$ and $A\left(D_{1}\right)=\left\{u_{1} u_{3}, u_{3} u_{1}\right\}$; let $D_{2}$ be the subgraph of $D$ with $V\left(D_{2}\right)=\left\{u_{1}, u_{2}, u_{3}, u_{4}\right\}$ and $A\left(D_{2}\right)=\left\{u_{1} u_{4}, u_{4} u_{1}, u_{2} u_{4}, u_{4} u_{2}, u_{2} u_{3}, u_{3} u_{2}\right\}$; for $3 \leq i \leq n-2$, let $D_{i}$ be the subgraph of $D$ with $V\left(D_{i}\right)=\left\{u_{1}, u_{2}, u_{i+2}\right\}$ and $A\left(D_{i}\right)=\left\{u_{1} u_{i+2}, u_{3} u_{i+2}, u_{i+2} u_{1}, u_{i+2} u_{3}\right\}$. Clearly, $\left\{D_{i} \mid 1 \leq i \leq n-2\right\}$ is a set of $n-$ 2 internally disjoint $S$-strong subgraphs, so $\kappa_{S}(D) \geq n-2$ and then $\kappa_{2}(D) \geq n-2$. By (3), we have $\kappa_{2}(D) \leq \min \left\{\delta^{+}(D), \delta^{-}(D)\right\}=n-2$. Hence, $\kappa_{2}(D)=n-2$. Let $e \in A(D)$; clearly $e$ must be incident with at least one vertex of $\left\{u_{i} \mid 1 \leq i \leq 2\lfloor n / 2\rfloor\right\}$. Then we have that $\kappa_{2}(D-e) \leq \min \left\{\delta^{+}(D-e), \delta^{-}(D-\right.$ $e)\}=n-3$ by (3). Hence, $D$ is minimally strong subgraph $(2, n-2)$-connected.

Now let $D$ be minimally strong subgraph $(2, n-2)$-connected. By Theorem 4.1, we have that $D \nsubseteq \overleftrightarrow{K}_{n}$, that is, $D$ can be obtained from a complete digraph $\overleftrightarrow{K}_{n}$ by deleting a nonempty arc set $M$. To end our argument, we need the following three claims. Let us start from a simple yet useful observation.

Proposition 5.4 No pair of arcs in M has a common head or tail.

Proof of Proposition 5.4. By (3) no pair of arcs in $M$ has a common head or tail, as otherwise we would have $\kappa_{2}(D) \leq n-3$.

Claim $2|M| \geq 3$.

Proof of Claim 2 Let $|M| \leq 2$. We may assume that $|M|=2$ as the case of $|M|=1$ can be considered in a similar and simpler way.

Let the arcs of $M$ have no common vertices; without loss of generality, $M=\left\{u_{1} u_{2}, u_{3} u_{4}\right\}$. Then $\kappa_{2}\left(D-u_{2} u_{1}\right)=n-2$ as $D-u_{2} u_{1}$ is a supergraph of $\overleftrightarrow{K}_{n}$ without a union of $\lfloor n / 2\rfloor$ vertex-disjoint 2-cycles including the cycles $u_{1} u_{2} u_{1}$ and $u_{3} u_{4} u_{3}$. Thus, $D$ is not minimally strong subgraph $(2, n-2)$-connected. Let the arcs of $M$ have no common vertex. By Proposition 5.4, without loss of generality, $M=\left\{u_{1} u_{2}, u_{2} u_{3}\right\}$. Then $\kappa_{2}\left(D-u_{3} u_{1}\right)=n-2$ as we showed in the beginning of the proof of this theorem. Thus, $D$ is not minimally strong subgraph $(2, n-2)$ connected. Now let the arcs of $M$ have the same vertices, i.e., without loss of generality, $M=\left\{u_{1} u_{2}, u_{2} u_{1}\right\}$. As above, $\kappa_{2}\left(D-u_{2} u_{1}\right)=n-2$ and $D$ is not minimally strong subgraph $(2, n-2)$-connected.

Claim 3 If $|M|=3$, then $\overleftrightarrow{K}_{n}[M]$ is a 3-cycle. 
Proof of Claim 3 Suppose that $D$ is minimally strong subgraph $(2, n-2)$ connected, but $\overleftrightarrow{K}_{n}[M]$ is not a 3-cycle. By Proposition 5.4, no pair of $\operatorname{arcs}$ in $M$ has a common head or tail. Thus, $\overleftrightarrow{K}_{n}[M]$ must be isomorphic to one of graphs in Figs. 3 and 4. If $\overleftrightarrow{K}_{n}[M]$ is isomorphic to one of graphs in Fig. 3, then $\kappa_{2}(D) \leq n-3$ by Claim 1 and so $D$ is not minimally strong subgraph $(2, n-2)$-connected, a contradiction. For an arc set $M_{0}$ such that $\overleftrightarrow{K}_{n}\left[M_{0}\right]$ is a union of $\lfloor n / 2\rfloor$ vertex-disjoint 2-cycles, by the argument before, we know that $\overleftrightarrow{K}_{n}-M_{0}$ is minimally strong subgraph $(2, n-2)$-connected. For the case that $\overleftrightarrow{K}_{n}[M]$ is isomorphic to $(a)$ or $(b)$ in Fig. 4, we have that $\overleftrightarrow{K}_{n}-M_{0}$ is a proper subgraph of $\overleftrightarrow{K}_{n}-M$, so $D=\overleftrightarrow{K}_{n}-M$ must not be minimally strong subgraph $(2, n-2)$-connected, this also produces a contradiction. Hence, the claim holds.

Claim 4 If $|M|>3$, then $\overleftrightarrow{K}_{n}[M]$ is a union of $\lfloor n / 2\rfloor$ vertex-disjoint 2-cycles.

Proof of Claim 4 Suppose that $D$ is minimally strong subgraph $(2, n-2)$ connected, but $\overleftrightarrow{K}_{n}[M]$ is not a union of $\lfloor n / 2\rfloor$ vertex-disjoint 2-cycles.

By Claim 1 and Proposition 4.3, we have that $\overleftrightarrow{K}_{n}[M]$ does not contain graphs in Fig. 3 as a subgraph. Then $\overleftrightarrow{K}_{n}[M]$ does not contain a path of length at least three. Hence, the underlying undirected graph of $M$ has at least two connectivity components. By the fact that if $M$ is a 3-cycle, then $\overleftrightarrow{K}_{n}-M$ is minimally strong subgraph $(2, n-2)$-connected, we conclude that $\overleftrightarrow{K}_{n}[M]$ does not contain a cycle of length three. By Claim $1, \overleftrightarrow{K}_{n}[M]$ does not contain a path of length two. By Proposition 5.4, no pair of arcs in $M$ has a common head or tail. Hence, each connectivity component of $\overleftrightarrow{K}_{n}[M]$ must be a 2-cycle or an arc. Since $D$ is minimally strong subgraph $(2, n-2)$-connected, no connectivity component of $\overleftrightarrow{K}_{n}[M]$ is an arc. We have arrived at a contradiction, proving Claim 4.

Hence, if a digraph $D$ is minimally strong subgraph $(2, n-2)$-connected, then $D \cong \overleftrightarrow{K}_{n}-M$, where $\overleftrightarrow{K}_{n}[M]$ is a cycle of order three or a union of $\lfloor n / 2\rfloor$ vertexdisjoint 2-cycles.

Now the claimed values of $F(n, 2, n-2)$ and $f(n, 2, n-2)$ can easily be verified.

Let $\mathfrak{F}(n, k, \ell)$ be the set of all minimally strong subgraph $(k, \ell)$-connected digraphs with order $n$. We define

Fig. 4 Two graphs for Claim 3

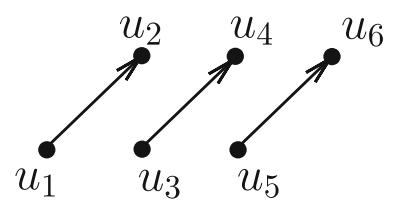

(a)

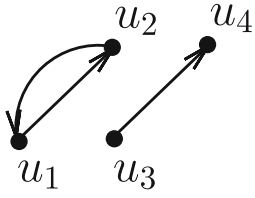

(b) 


$$
F(n, k, \ell)=\max \{|A(D)| \mid D \in \mathfrak{F}(n, k, \ell)\}
$$

and

$$
f(n, k, \ell)=\min \{|A(D)| \mid D \in \mathfrak{F}(n, k, \ell)\} .
$$

We further define

$$
\operatorname{Ex}(n, k, \ell)=\{D|D \in \mathfrak{F}(n, k, \ell),| A(D) \mid=F(n, k, \ell)\}
$$

and

$$
e x(n, k, \ell)=\{D|D \in \mathfrak{F}(n, k, \ell),| A(D) \mid=f(n, k, \ell)\} .
$$

Note that Theorem 5.3 implies that $\operatorname{Ex}(n, 2, n-2)=\left\{\overleftrightarrow{K_{n}}-M\right\}$ where $M$ is an arc set such that $\overleftrightarrow{K}_{n}[M]$ is a directed 3-cycle, and $\operatorname{ex}(n, 2, n-1)=\left\{\overleftrightarrow{K}_{n}-M\right\}$ where $M$ is an arc set such that $\overleftrightarrow{K}_{n}[M]$ is a union of $\lfloor n / 2\rfloor$ vertex-disjoint directed 2-cycles.

The following result concerns a sharp lower bound for the parameter $f(n, k, \ell)$.

Theorem 5.5 For $2 \leq k \leq n$, we have

$$
f(n, k, \ell) \geq n \ell \text {. }
$$

Moreover, the following assertions hold: ( $i)$ If $\ell=1$, then $f(n, k, \ell)=n$; (ii) If $2 \leq \ell \leq n-1$, then $f(n, n, \ell)=n \ell$ for $k=n \notin\{4,6\}$; (iii) If $n$ is even and $\ell=n-2$, then $f(n, 2, \ell)=n \ell$.

Proof By (3), for all digraphs $D$ and $k \geq 2$ we have $\kappa_{k}(D) \leq \delta^{+}(D)$ and $\kappa_{k}(D) \leq \delta^{-}(D)$. Hence for each $D$ with $\kappa_{k}(D)=\ell$, we have that $\delta^{+}(D), \delta^{-}(D) \geq \ell$, so $|A(D)| \geq n \ell$ and then $f(n, k, \ell) \geq n \ell$.

For the case that $\ell=1$, let $D$ be a dicycle $\overrightarrow{C_{n}}$. Clearly, $D$ is minimally strong subgraph $(k, 1)$-connected, and we know $|A(D)|=n$, so $f(n, k, 1)=n$.

For the case that $k=n \notin\{4,6\}$ and $2 \leq \ell \leq n-1$, let $D \cong \overleftrightarrow{K_{n}}$. By Theorem 2.2, $D$ can be decomposed into $n-1$ Hamiltonian cycles $H_{i}(1 \leq i \leq n-1)$. Let $D_{\ell}$ be the spanning subgraph of $D$ with arc sets $A\left(D_{\ell}\right)=\bigcup_{1 \leq i \leq \ell} A\left(H_{i}\right)$. Clearly, we have $\kappa_{n}\left(D_{\ell}\right) \geq \ell$ for $2 \leq \ell \leq n-1$. Furthermore, by (3), we have $\kappa_{n}\left(D_{\ell}\right) \leq \ell$ since the indegree and out-degree of each vertex in $D_{\ell}$ are both $\ell$. Hence, $\kappa_{n}\left(D_{\ell}\right)=\ell$ for $2 \leq \ell \leq n-1$. For any $e \in A\left(D_{\ell}\right)$, we have $\delta^{+}\left(D_{\ell}-e\right)=\delta^{-}\left(D_{\ell}-e\right)=\ell-1$, so $\kappa_{n}\left(D_{\ell}-e\right) \leq \ell-1$ by (3). Thus, $D_{\ell}$ is minimally strong subgraph $(n, \ell)$-connected. As $\left|A\left(D_{\ell}\right)\right|=n \ell$, we have $f(n, n, \ell) \leq n \ell$. From the lower bound that $f(n, k, \ell) \geq n \ell$, we have $f(n, n, \ell)=n \ell$ for the case that $2 \leq \ell \leq n-1, n \notin\{4,6\}$.

Part (iii) follows directly from Theorem 5.3.

To prove two upper bounds on the number of arcs in a minimally strong subgraph $(k, \ell)$-connected digraph, we will use the following result from [2].

Theorem 5.6 Every strong digraph D on n vertices has a strong spanning subgraph $H$ with at most $2 n-2$ arcs and equality holds only if $H$ is a symmetric digraph whose underlying undirected graph is a tree. 
Proposition 5.7 We have (i) $F(n, n, \ell) \leq 2 \ell(n-1)$; (ii) For every $k(2 \leq k \leq n)$, $F(n, k, 1)=2(n-1)$ and $\operatorname{Ex}(n, k, 1)$ consists of symmetric digraphs whose underlying undirected graphs are trees.

Proof $(i)$ Let $D=(V, A)$ be a minimally strong subgraph $(n, \ell)$-connected digraph, and let $D_{1}, \ldots, D_{\ell}$ be arc-disjoint strong spanning subgraphs of $D$. Since $D$ is minimally strong subgraph $(n, \ell)$-connected and $D_{1}, \ldots, D_{\ell}$ are pairwise arc-disjoint, $|A|=\sum_{i=1}^{\ell}\left|A\left(D_{i}\right)\right|$. Thus, by Theorem 5.6, $|A| \leq 2 \ell(n-1)$.

(ii) In the proof of Proposition 5.2 we showed that a digraph $D$ is strong if and only if $\kappa_{k}(D) \geq 1$. Now let $\kappa_{k}(D) \geq 1$ and a digraph $D$ has a minimal number of arcs. By Theorem 5.6, we have that $|A(D)| \leq 2(n-1)$ and if $D \in \operatorname{Ex}(n, k, 1)$ then $|A(D)|=2(n-1)$ and $D$ is a symmetric digraph whose underlying undirected graph is a tree.

We now study the minimally strong subgraph $(k, \ell)$-arc-connected digraphs. By Proposition 4.8 and Theorem 4.5, we have the following result.

Proposition 5.8 The following assertions hold:

A digraph $D$ is minimally strong subgraph

$$
\text { -arc-connected if and only if } D \text { is minimally strong digraph; }
$$

Let $2 \leq k \leq n$. If $k \notin\{4,6\}$, or, $k \in\{4,6\}$ and $k<n$, then a digraph $D$ is minimally strong subgraph $(k, n-1)$-arc-connected if and only if $D \cong \overleftrightarrow{K}_{n}$.

The following result characterizes minimally strong subgraph $(2, n-2)$-arcconnected digraphs. This characterization is different from the characterization of minimally strong subgraph $(2, n-2)$-connected digraphs obtained in Theorem 5.3.

Theorem 5.9 A digraph D is minimally strong $\operatorname{subgraph}(2, n-2)$-arc-connected if and only if $D$ is a digraph obtained from the complete digraph $\overleftrightarrow{K}_{n}$ by deleting an arc set $M$ such that $\overleftrightarrow{K}_{n}[M]$ is a union of vertex-disjoint cycles which cover all but at most one vertex of $\overleftrightarrow{K}_{n}$

Proof Let $D$ be a digraph obtained from the complete digraph $\overleftrightarrow{K}_{n}$ by deleting an arc set $M$ such that $\overleftrightarrow{K}_{n}[M]$ is a union of vertex-disjoint cycles which cover all but at most one vertex of $\overleftrightarrow{K}_{n}$. To prove the theorem it suffices to show that (a) $D$ is minimally strong subgraph $(2, n-2)$-arc-connected, that is, $\lambda_{2}(D) \geq n-2$ but for any arc $e \in A(D), \lambda_{2}(D-e) \leq n-3$, and (b) if a digraph $H$ minimally strong subgraph $(2, n-2)$-arc-connected then it must be constructed from $\overleftrightarrow{K}_{n}$ as the digraph $D$ above. Thus, the remainder of the proof has two parts.

Part (a). We just consider the case that $\overleftrightarrow{K}_{n}[M]$ is a union of vertex-disjoint cycles which cover all vertices of $\overleftrightarrow{K}_{n}$, since the argument for the other case is similar. For any $e \in A\left(\overleftrightarrow{K}_{n}\right) \backslash M$, we know $e$ must be adjacent to at least one element of $M$, so $\lambda_{2}(D-e) \leq \min \left\{\delta^{+}(D-e), \delta^{-}(D-e)\right\}=n-3$ by (3). Hence, it suffices to show that $\lambda_{2}(D)=n-2$ in the following. We clearly have that $\lambda_{2}(D) \leq n-2$ by (3), so 
we will show that for $S=\{x, y\} \subseteq V(D)$, there are at least $n-2$ arc-disjoint $S$ strong subgraphs in $D$.

Case 1. $x$ and $y$ belong to distinct cycles of $\overleftrightarrow{K}_{n}[M]$. We just consider the case that the lengths of these two cycles are both at least three, since the arguments for the other cases are similar. Assume that $u_{1} x, x u_{2}$ belong to one cycle, and $u_{3} y, y u_{4}$ belong to the other cycle. Note that $u_{1} u_{2}, u_{3} u_{4} \in A(D)$ since the lengths of these two cycles are both at least three.

Let $D_{1}$ be the 2-cycle $x y x$; let $D_{2}$ be the subgraph of $D$ with vertex set $\left\{x, y, u_{1}, u_{2}\right\}$ and arc set $\left\{x u_{1}, u_{1} u_{2}, u_{2} x, y u_{2}, u_{2} y\right\}$; let $D_{3}$ be the subgraph of $D$ with vertex set $\left\{x, y, u_{3}, u_{4}\right\}$ and arc set $\left\{y u_{3}, u_{3} u_{4}, u_{4} y, x u_{3}, u_{3} x\right\}$; let $D_{4}$ be the subgraph of $D$ with vertex set $\left\{x, y, u_{1}, u_{4}\right\}$ and arc set $\left\{x u_{4}, u_{4} x, y u_{1}, u_{1} y, u_{1} u_{4}, u_{4} u_{1}\right\}$; for each vertex $u \in V(D) \backslash\left\{x, y, u_{1}, u_{2}, u_{3}, u_{4}\right\}$, let $D_{u}$ be a subgraph of $D$ with vertex set $\{u, x, y\}$ and arc set $\{u x, x u, u y, y u\}$. It is not hard to check that these $n-2 S$-strong subgraphs are arc-disjoint.

Case 2. $x$ and $y$ belong to the same cycle, say $u_{1} u_{2} \cdots u_{t} u_{1}$, of $\overleftrightarrow{K}_{n}[M]$. We just consider the case that the length of this cycle is at least three, since the argument for the remaining case is simpler.

Subcase 2.1. $x$ and $y$ are adjacent in the cycle. Without loss of generality, let $x=u_{1}, y=u_{2}$. Let $D_{1}$ be the subgraph of $D$ with vertex set $\left\{x, y, u_{3}\right\}$ and arc set $\left\{y x, x u_{3}, u_{3} y\right\}$; let $D_{2}$ be the subgraph of $D$ with vertex set $\left\{x, y, u_{3}, u_{t}\right\}$ and arc set $\left\{u_{3} x, x u_{t}, u_{t} u_{3}, u_{t} y, y u_{t}\right\}$; for each vertex $u \in V(D) \backslash\left\{x, y, u_{3}, u_{t}\right\}$, let $D_{u}$ be a subgraph of $D$ with vertex set $\{u, x, y\}$ and arc set $\{u x, x u, u y, y u\}$. It is not hard to check that these $n-2 S$-strong subgraphs are arc-disjoint.

Subcase 2.2. $x$ and $y$ are nonadjacent in the cycle. Without loss of generality, let $x=u_{1}, y=u_{3}$. Let $D_{1}$ be the 2-cycle $x y x$; let $D_{2}$ be the subgraph of $D$ with vertex set $\left\{x, y, u_{2}, u_{t}\right\}$ and arc set $\left\{y u_{2}, u_{2} x, x u_{t}, u_{t} y\right\}$; for each vertex $u \in V(D) \backslash\left\{x, y, u_{2}, u_{t}\right\}$, let $D_{u}$ be a subgraph of $D$ with vertex set $\{u, x, y\}$ and arc set $\{u x, x u, u y, y u\}$. It is not hard to check that these $n-2 S$-strong subgraphs are arc-disjoint.

Part (b). Let $H$ be minimally strong subgraph $(2, n-2)$-arc-connected. By Lemma 4.4, we have that $H \nsubseteq \overleftrightarrow{K}_{n}$, that is, $H$ can be obtained from a complete digraph $\overleftrightarrow{K}_{n}$ by deleting a nonempty arc set $M$. To end our argument, we need the following claim. Let us start from a simple yet useful observation, which follows by Inequality (3)

Proposition 5.10 No pair of arcs in $M$ has a common head or tail.

Thus, $\overleftrightarrow{K}_{n}[M]$ must be a union of vertex-disjoint cycles or paths, otherwise, there are two $\operatorname{arcs}$ of $M$ such that they have a common head or tail, a contradiction with Proposition 5.10.

Claim $1 \overleftrightarrow{K}_{n}[M]$ does not contain a path of order at least two.

Proof of Claim 1 Let $M^{\prime} \supseteq M$ be a set of arcs obtained from $M$ by adding some arcs from $\overleftrightarrow{K}_{n}$ such that the digraph $\overleftrightarrow{K}_{n}\left[M^{\prime}\right]$ contains no path of order at least two. Note 
that $\overleftrightarrow{K}_{n}\left[M^{\prime}\right]$ is a supergraph of $\overleftrightarrow{K}_{n}[M]$ and is a union of vertex-disjoint cycles which cover all but at most one vertex of $\overleftrightarrow{K}_{n}$. By Part (a), we have that $\lambda_{2}\left(\overleftrightarrow{K}_{n}\left[M^{\prime}\right]\right)=n-2$, so $\overleftrightarrow{K}_{n}[M]$ is not minimally strong subgraph $(2, n-2)$-arcconnected, a contradiction.

It follows from Claim 1 and its proof that $\overleftrightarrow{K}_{n}[M]$ must be a union of vertexdisjoint cycles which cover all but at most one vertex of $\overleftrightarrow{K}_{n}$, which completes the proof of Part (b).

\section{Discussion}

Corollaries 3.5 and 3.7 shed some light on the complexity of deciding, for fixed $k, \ell \geq 2$, whether $\lambda_{k}(D) \geq \ell$ for semicomplete and symmetric digraphs $D$. However, it is unclear what is the complexity above for every fixed $k, \ell \geq 2$. If Conjecture 1 is correct, then the $\lambda_{k}(D) \geq \ell$ problem can be solved in polynomial time for semicomplete digraphs. However, Conjecture 1 seems to be very difficult. It was proved in [13] that for fixed $k, \ell \geq 2$ the problem of deciding whether $\kappa_{k}(D) \geq \ell$ is polynomial-time solvable for both semicomplete and symmetric digraphs, but it appears that the approaches to prove the two results cannot be used for $\lambda_{k}(D)$. Some well-known results such as the fact that the hamiltonicity problem is NP-complete for undirected 3-regular graphs, indicate that the $\lambda_{k}(D) \geq \ell$ problem for symmetric digraphs may be NP-complete, too.

One of the most interesting results of this paper is the characterization of minimally strong subgraph $(2, n-2)$-connected digraphs. As a simple consequence of the characterization, we can determine the values of $f(n, 2, n-2)$ and $F(n, 2, n-2)$. It would be interesting to determine $f(n, k, n-2)$ and $F(n, k, n-$ 2 ) for every value of $k \geq 3$. (Obtaining characterizations of all $(k, n-2)$-connected digraphs for $k \geq 3$ seems a very difficult problem.) It would also be interesting to find a sharp upper bound for $F(n, k, \ell)$ for all $k \geq 2$ and $\ell \geq 2$.

Acknowledgements We are thankful to Anders Yeo for discussions related to the complexity of computing strong subgraph $(k, \ell)$-arc-connectivity for semicomplete and symmetric digraphs

Funding Yuefang Sun was supported by Zhejiang Provincial Natural Science Foundation (no. LY20A010013). Gregory Gutin was partially supported by Royal Society Wolfson Research Merit Award.

\section{Availability of data and material Not applicable}

\section{Declarations}

Conflicts of interest All author(s) declare that they have no conflicts of interest.

Code availability Not applicable.

Open Access This article is licensed under a Creative Commons Attribution 4.0 International License, which permits use, sharing, adaptation, distribution and reproduction in any medium or format, as long as you give appropriate credit to the original author(s) and the source, provide a link to the Creative 
Commons licence, and indicate if changes were made. The images or other third party material in this article are included in the article's Creative Commons licence, unless indicated otherwise in a credit line to the material. If material is not included in the article's Creative Commons licence and your intended use is not permitted by statutory regulation or exceeds the permitted use, you will need to obtain permission directly from the copyright holder. To view a copy of this licence, visit http:// creativecommons.org/licenses/by/4.0/.

\section{References}

1. Aouchiche, M., Hansen, P.: A survey of Nordhaus-Gaddum type relations. Discrete Appl. Math. 161(4/5), 466-546 (2013)

2. Bang-Jensen, J., Gutin, G.: Digraphs: Theory, Algorithms and Applications, 2nd edn. Springer, London (2009)

3. Bang-Jensen, J., Gutin, G., Yeo, A.: Arc-disjoint strong spanning subdigraphs of semicomplete compositions. J. Graph Theory 95(2), 267-289 (2020)

4. Bang-Jensen, J., Huang, J.: Decomposing locally semicomplete digraphs into strong spanning subdigraphs. J. Combin. Theory Ser. B 102, 701-714 (2012)

5. Bang-Jensen, J., Yeo, A.: Decomposing $k$-arc-strong tournaments into strong spanning subdigraphs. Combinatorica 24, 331-349 (2004)

6. Boesch, F., Tindell, R.: Robbins' theorem for mixed multigraphs. Am. Math. Mon. 87, 716-719 (1980)

7. Chudnovsky, M., Scott, A., Seymour, P.D.: Disjoint paths in unions of tournaments. J. Combin. Theory Ser. B 135, 238-255 (2019)

8. Hager, M.: Pendant tree-connectivity. J. Combin. Theory Ser. B 38, 179-189 (1985)

9. Li, X., Mao, Y.: Generalized Connectivity of Graphs. Springer, Switzerland (2016)

10. Palmer, E.M.: On the spanning tree packing number of a graph: a survey. Discrete Math. 230, 13-21 (2001)

11. Shiloach, Y.: Edge-disjoint branching in directed multigraphs. Inf. Process. Lett. 8(1), 24-27 (1979)

12. Sun, Y., Gutin, G., Ai, J.: Arc-disjoint strong spanning subdigraphs in compositions and products of digraphs. Discrete Math. 342(8), 2297-2305 (2019)

13. Sun, Y., Gutin, G., Yeo, A., Zhang, X.: Strong subgraph $k$-connectivity. J. Graph Theory 92(1), 5-18 (2019)

14. Thomassen, C.: Highly connected non-2-linked digraphs. Combinatorica 11(4), 393-395 (1991)

15. Tillson, T.W.: A Hamiltonian decomposition of $K_{2 m}^{*}, 2 m \geq 8$. J. Combin. Theory Ser. B 29(1), 68-74 (1980)

Publisher's Note Springer Nature remains neutral with regard to jurisdictional claims in published maps and institutional affiliations. 